\title{
REVIEW
}

\section{Jun: the master regulator in healthy and cancer cells}

\author{
Petr Vaňhara, Jan Šmarda \\ Laboratory of Cell Differentiation, Institute of Experimental Biology, Faculty of Science, Masaryk University, \\ Brno, Brno, Czech Republic
}

Received $25^{\text {th }}$ September 2006.

Revised $5^{\text {th }}$ October 2006.

Published online $1^{\text {st }}$ December 2006.

\begin{abstract}
Summary
Healthy cells strictly regulate gene transcription to control crucial cellular regulatory pathways. Members of the Jun protein family, c-Jun, JunB, and JunD are key subunits of the transcription factor AP-1 that controls transcription from various gene promoters. The genes targeted by Jun affect essential life processes, such as cell cycle progression, differentiation or programmed cell death. Therefore, the loss of proper Jun function is often associated with cancer. This review summarizes recent advances in understanding of function of the Jun proteins in healthy and cancer cells.
\end{abstract}

Keywords: AP-1 - Jun - cancer - apoptosis - cell cycle - differentiation

\section{INTRODUCTION}

The Jun proteins are members or the basic leucine zipper (bZIP) protein family that participate in the formation of the Activation Protein 1 (AP-1) transcription factor. To form the AP-1, the Jun moiety (c-Jun, JunB or JunD) dimerizes with one of the Fos proteins (c-Fos, FosB, Fra1, Fra2) or one of the CREB/ATF/Maf proteins. Some members of the Jun family can also bind the other proteins possessing dimerization motifs, such as PU.1 or

Jan Šmarda, Laboratory of Cell Differentiation, Institute of Experimental Biology, Faculty of Science, Masaryk University, Brno, Kotlářská 2, 61137 Brno, Czech Republic

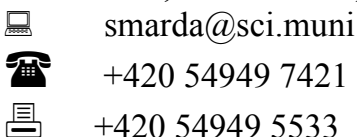

县 $\quad+420549495533$
GATA-1 (Liew et al. 2006), the master transcriptional regulators of hematopoiesis. The composition of AP-1 determines its DNA-binding specifity (Kouzarides and Ziff 1989). AP-1 often binds to the phorbol 12-O-tetradecanoyl-13-acetate (TPA)-responsive elements (TRE, 5'TGACTCA3') or cAMP-responsive elements (CRE, 5'TGACGTCA-3') (Lee et al. 1987, Inagaki et al. 1992). TREs and CREs are present in promoters of many genes coding for collagenase I, metallothionein $\mathrm{II}_{\mathrm{A}}$, interleukin 2, fibronectin, vimentin, cell cycle regulators and many others (Rahmsdorf 1996, Karin et al. 1997, Rinehart-Kim et al. 2000, Angel et al. 2001, Jochum et al. 2001, Shaulian and Karin 2001). Moreover, there is frequent cross-talk between the Jun proteins and other transcription factors, such as pRB (Nead et al. 1998), ATF (Tsai et al. 1996), CREB (Benbrook and Jones 1990), NFKB, NFAT (Macian et al. 2001) or the homeodomain proteins. These intermolecular interactions extend the effects of AP-1 to 
cellular signalling pathways driven by various ligand-activated receptors such as receptors for glucocorticoid hormone, estrogen hormone, retinoic acid, vitamin D3 and MyoD (Gaub et al. 1990, Jonat et al. 1990, Schüle et al. 1990, Bengal et al. 1992). Therefore, the Jun proteins integrate complex information from cellular environment and connect it with executive molecular network to optimize the cell reponse.

\section{PROLIFERATION AND PROGRAMMED CELL DEATH}

The c-Jun protein is essential for control of the cell cycle. It induces expression of cyclin D1, the positive regulator of the G1-phase cyclin-dependent kinases CDK4/CDK6. Growth of fibroblasts derived from c-jun-deficient or haploinsufficient mice is arrested and this effect is not reversible by mitogens (Johnson et al. 1993, Eferl et al. 1999, Schreiber et al. 1999). The lack of c-Jun results in the p53-mediated inhibition of the S-phase-specific CDKs by $\mathrm{p} 21^{\text {cip/kip }}$ (Schreiber et al. 1999). Hepatoblasts derived from the c-Jun ${ }^{-/-}$mice are growth-arrested as well. Therefore, upon partial hepatoctomy the c-Jun-deficient mouse hepatoblasts cannot regenerate liver (Behrens et al. 2002).

In contrast to the growth enhancing properties of c-Jun, the JunB and JunD proteins frequently function in the opposite way (Castellazzi et al. 1991). The JunB protein induces cell cycle arrest by activating the transcription of $\mathrm{p} 16^{\text {ink4a }}$, the CDK inhibitor (Passegué and Wagner 2000). The p16 $6^{\text {ink4a }}$ protein suppresses the growth of mouse 3T3 fibroblasts in the G1 phase of the cell cycle and induces premature senescence. In addition, the JunB protein inhibits phosphorylation of the $\mathrm{pRb}$ tumor suppressor by recruiting cyclin D1-CDK4/6 complexes. Inhibition of JunB in myeloid progenitor cells in vivo induces leukemia-like diseases typified by the accumulation of neutrophil granulocytes (Passegué et al. 2001). Similarly to JunB, the JunD also acts as a growth suppressor. The over-expression of JunD arrests the growth of immortalized fibroblasts and JunD-deficiency stimulates proliferation of these cells (Pfarr et al. 1994, Weitzman et al. 2000). Growth arrest induced by JunD is mediated by $\mathrm{p} 53$ and $\mathrm{p} 119^{\mathrm{ARF}}$ proteins (Weitzman et al. 2000).

The role of c-Jun in the regulation of programmed cell death is tissue-specific: it stimulates self-destruction in some cells, while suppressing it in the others. The pro-apoptotic effects of c-Jun are documented in primary sympathetic neurons (Kanamoto et al. 2000). Inhibition of c-Jun by its dominant negative mutants suppresses apoptosis from occurring upon withdrawal of the neural growth factor (Estus et al.
1994, Ham et al. 1995, Behrens et al. 1999). The cJun protein is also required for induction of the apoptosis of 3T3 fibroblasts by alkylating agents (Kolbus et al. 2000). Serine to alanine substitutions at positions 63 and 73 of the c-Jun protein rescue primary murine cerebellar granule cells from kainate-induced apoptosis (Cheung et al. 1998). In contrast, the c-Jun protein does not control programmed cell death in early developing tissues: the c-Jun-deficient mouse embryos possess normal frequency of apoptosis at 11.5 days post coitum (dpc) (Roffler-Tarlov et al. 1996). Interestingly, at E13 dpc the frequency of apoptosis dramatically increases and the c-Jun ${ }^{-/-}$mice die because of the massive cell death of hepatoblast and liver hematopoetic cells (Eferl et al. 1999). Experiments performed on various cell lines showed that the function of the c-Jun protein in the control of programmed cell death varies according to the cellular or microenvironmental context. Overexpression of c-Jun induces cell death rather than differentiation of murine MEL erythroleukemia cells (Poindessous-Jazat et al. 2002), while the same protein enhances the sensitivity of v-mybtransformed BM2 monoblasts to differentiationpromoting agents such as phorbol esters or retinoic acid without any apparent effect on the frequency of apoptosis (Ševčíková et al. 2002).

The JunD and JunB proteins also participate in the control of programmed cell death machinery, although using different molecular mechanisms. While JunD suppresses cell death in a p53dependent manner (Hilfiker-Kleiner et al. 2005, Ricci et al. 2005), JunB activates apoptosis by controlling the expression of anti-apoptotic genes $b c l-2$ and $b c l_{\mathrm{xl}}$ (Passegué et al. 2001). In addition, JunB mediates apoptosis caused by bile acids (Bernt et al. 2006). Steroid bile acids are potent activators of ERK1/2 and JNK1/2 pathways, thus enhancing the activity of the Jun proteins. According to signals transduced by these pathways, the Jun proteins determine whether the cell starts the process of self-destruction or not (Quiao et al. 2003).

\section{TISSUE DEVELOPMENT AND REPAIR}

The role of the Jun proteins in organ development is also tissue-specific. The c-jun-knock-out mice are not viable due to failure of liver formation. Absence of the c-Jun protein from embryonic stem cells and hepatoblasts is lethal for liver development due to the increased rate of apoptosis and necrosis (Hilberg et al. 1993). Furthermore, disorders of liver development caused by c-Jun-deficiency impair embryonal hematopoiesis (Eferl et al. 1999). The lack of the c-Jun protein also affects the development of the cardiovascular system. The malformations of aorta ascendens and pulmonary 
artery occurring in c-jun ${ }^{-/-}$mice are similar to the abnormal positioning of the aortic arch and the wide connection between right and left ventricles described in humans with persistent truncus arteriosus (Rossant et al. 1996, Eferl et al. 1999). This disease can result from either improper crosstalk between the c-Jun and Pax3 transcription factors (Wiggan et al. 2002) or deregulation of vascular endothelial growth factors (VEGF) by AP1 (Naruishi et al. 2003).

As with the c-jun knock-out mice, development of JunB-deficient mice is aborted between 8.5 and $10.0 \mathrm{dpc}$. These mice die from avascularisation of extraembryonic tissues (Schorpp-Kistner et al. 1999). Disorder of the vasal tissues in these mice presumably results from the incapacity of chondroallantoic plate vessels to expand in trophoblasts. Interestingly, while c-Jun and JunB act as mutual antagonists in a variety of tissues, they possess similar effects on vascular development. JunB also controls hematopoiesis by acting as a negative regulator of proliferation of hematopoetic stem cells (HSC). Inactivation of JunB deregulates expansion of the HSC, thus inducing myeloid leukemia in mice (Passégue et al. 2001). In addition, the JunB protein participates in the control of differentiation. The ectopic expression of JunB up-regulates several differentiation-promoting cytokines in $\mathrm{T}$ lymphocytes. The JunB protein is also expressed in epidermal and endodermal gut epithelial cells undergoing terminal differentiation (Wilkinson et al. 1989, Szabowski et al. 2001).

In contrast to c-Jun and JunB, a deficiency in JunD does not result in abortion of mouse development and the $\mathrm{JunD}^{-/-}$mice are viable. However, they exhibit impaired growth, hormone disbalance and reproduction defects because of weak spermatogenesis (Thepot et al. 2000). Recently, the group of Ricci indicated that the JunD protein is involved in the response of heart cells to mechanical stress, because the JunD-deficient mice exhibit spontaneous cardiomyopathy and an increased rate of apoptosis upon mechanical pressure (Ricci et al. 2005).

The role of Jun proteins in tissue development is also important in wound healing. The Jun proteins are up-regulated in a body suffering from injury or ischemia, thus activating transcription of various genes involved in tissue repair, such as genes coding for matrix metalloproteases. When inactivated, wound healing is delayed and disharmonised (Shirai et al. 2001, Florin et al. 2004, 2006, Hilfhiker et al. 2005).

\section{CARCINOGENESIS}

The border between physiological cell expansion during wound healing and deregulated cancer outgrowth is remarkably narrow. Therefore, deregulation of the Jun proteins that are involved in the control of tissue repair can contribute to carcinogenesis. There is an oncogenic version of c$j u n$, the v-jun that has been identified as one of the first oncogenes. v-jun oncogene was isolated from acutely transforming retrovirus (avian sarcoma virus 17) and provided the first clue that AP-1 is deeply involved in carcinogenesis. Later, various membrane-associated- or cytoplasmic oncoproteins, such as Ras, Src and Raf were shown to up-regulate AP-1 and this effect was partially reponsible for their transforming functions (Schütte et al. 1989).

The v-Jun oncoprotein differs from its cellular homologue, the c-Jun, by 27 -amino acid truncation in the amino-terminal transactivation domain and two nonconservative amino-acid substitutions located in the carboxy-terminal domain involved in dimerization and DNA-binding (Nishimura and Vogt 1988). The N-terminal truncation prevents phosphorylation of the Jun protein by Jun Nterminal kinase (JNK) (Hibi et al. 1993), thus controlling its transactivating capability. V-Jun induces fibrosarcomas in chickens by enhancing the proliferation rate of fibroblasts in damaged tissues (Marshall et al. 1992). Similarly, transgenic mice over-expressing v-Jun also develop dermal fibrosarcomas and exhibit abnormal wound repair (Schuh et al. 1990, Marshal et al. 1993). These effects may result from the $v$-Jun-induced deregulation of either MAPK/ERK signalling pathways and/or the cell cycle regulators, such as p21 $1^{\text {cip }}$ (Black et al. 2002, MacLaren et al. 2003). Hence, the v-Jun protein can alter cellular physiology at multiple levels to start tumor formation.

The role of c-Jun in carcinogenesis is remarkably diverse and depends on multiple factors. The position of the c-Jun protein in the centre of the cellular signalling network predetermines this protein for the regulation of many cellular processes. Suppression of AP-1 activity was shown to disturb the cell cycle progression of cultured cells and to reduce the efficiency of oncoprotein-mediated cell transformation (Lloyd et al. 1991, Johnson et al. 1996). Phosphorylations of Ser 63 and Ser 73 residues of the c-Jun protein enhance its transformation activity (Smeal et al. 1991, Smeal et al. 1992, Behrens et al. 2000), but over-expression of this protein in cells does not cause tumor formation (Grigoriadis et al. 1993). c-Jun cooperates with the c-Fos protein in the formation of osteosarcomas (Wang et al. 1995). In addition to the deregulation of proliferation and differentiation, c-Jun and JunB participate in malignant transformation by interfering with signal transduction pathways from cell surface molecules, such as integrins and cadherins, to intracellular effectors. Weaker cell adhesion can enhance the formation of metastases. Therefore, ectopic 
expression of the JAC gene that is targeted by the cJun protein induces anchorage-independent growth of avian fibroblasts (Hartl et al. 2001). Similarly, the c-Jun and JunB proteins can also induce the anchorage-independent growth of Rat1 fibroblasts
(Leaner et al. 2003). Mammary epithelial cells over-expressing c-Jun lose polarity but they do not undergo malignant transformation (Fialka et al. 1996).

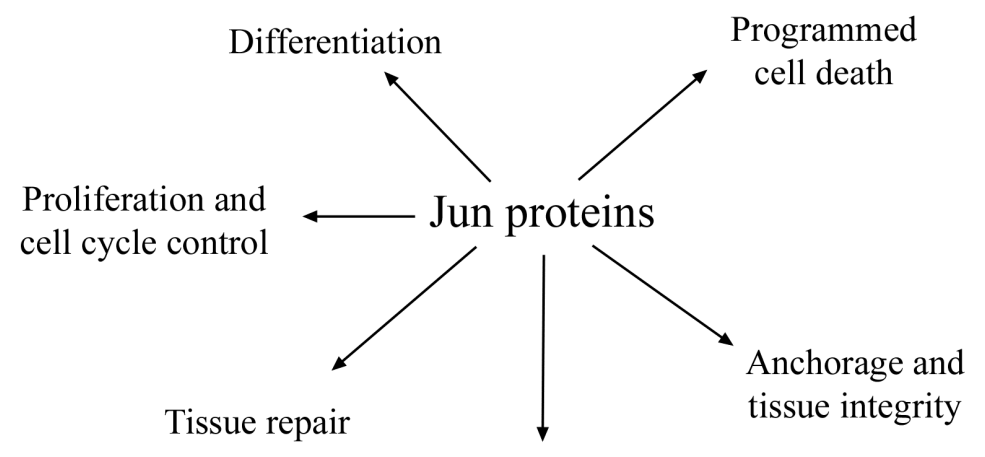

Cancer

Fig. 1. Functions of the Jun proteins

While alteration of the c-jun expression is associated with early transformation events in human lung cancer (Szabo et al. 1996), its upregulation can promote the anchorage-independent growth of the human bronchial epithelial cell line BEAS-2B in semi-solid media and therefore contribute to the formation of metastases in vivo (Maeno et al. 2006). c-Jun is also associated with multistage cancer development in an AP-1independent manner (Watts et al. 1995). The c-Jun protein suppresses the anti-proliferative effects of retinoic acid in breast cancer cells (Yang et al. 1997). c-Jun is also involved in the development of leukemias. c-jun expression is activated by the AML1-ETO protein chimera in the bone marrow of patients with acute myeloid leukemia (AML) (Elsasser et al. 2003).

The c-Jun protein then binds to and inhibits $\mathrm{C} / \mathrm{EBP} \alpha$, thus blocking differentiation of bone marrow cells (Rangatia et al. 2003). The Bcr-Abl protein chimera marking chronic myeloid leukemia induces phosphorylation of c-Jun by activating the Jun N-terminal kinase JNK. Hyperphosphorylated c-Jun decreases the sensitivity of the cell to the absence of growth factors. Inhibition of c-Jun by its dominant negative mutants suppresses the transformation induced by Bcr-Abl in murine myeloid cells (Raitano et al 1995). On the other hand, c-jun expression increases during the dexomethasone-induced apoptosis of human leukemic lymphoblasts, while leaving the levels of JunD or JunB unaffected (Zhou et al 1996).

The introduction of dominant negative mutants brought new perspectives to the analysis of the Jun function. The dominant negative jun mutants became promising tools for therapy of various types of cancer (Yasumoto et al. 2001, Suto et al. 2004). Neyns et al 1999 described significant inhibition of the outgrowth of colonies of human AZ224, SKOV3 and OVCAR3 ovarian cancer cells by transient expression of a dominant negative c-jun mutant (TAM67). Similarly, the TAM67 inhibited tumor promotion in the epidermis (Young et al. 1999), reduced the number of epidermal tumours induced by UV in the skin of hairless mice (Cooper et al. 2003) and decreased the growth rate of malignant breast cell lines (Ludes-Meyers et al. 2001, Liu et al. 2002). Inhibition of c-Jun also reduced the propagation of malignant mouse epidermal JB6 cells resulting from alterations of matrix-metalloproteinases (Dong et al. 1997).

In contrast to c-Jun, the JunD and JunB proteins act rather as tumour suppressors. The JunB suppresses activity of the AP-1 complex, thus inhibiting proliferation of malignant mouse keratinocytes upon $\gamma$-irradiation (Finch et al. 2002). Inactivation of the $j u n B$ gene by methylation can contribute to the development of certain types of chronic myeloid leukemia.

\section{CONCLUSION}

The Jun proteins are important signalling molecules that regulate the fate of a cell by controlling essential processes, such as cell cycle progression, execution of programmed cell death or differentiation (Fig. 1). These proteins can perform 
various functions according to the cellular/tissue context or developmental stage. Deregulation or absence of the Jun proteins from cells can contribute to malignant transformation. Therefore, the Jun proteins are suitable markers of tumour diseases as well as targets for gene therapy.

\section{ACKNOWLEDGEMENTS}

This work was supported by grant 301/06/0036 (JŠ) of the Grant Agency of the Czech Republic, MSM0021622415 (JŠ) and FRVŠ 111/2006 (PV) of the Ministry of Education, Youth and Sports of the Czech Republic.

\section{REFERENCES}

Angel P., Szabowski A., Schorpp-Kistner M.: Function and regulation of AP-1 subunits in skin physiology and pathology. Oncogene 20: 2413-2423, 2001.

Behrens A., Jochum W., Sibilia M. et al.: Oncogenic transformation by ras and fos is mediated by c-Jun N-terminal phosphorylation. Oncogene 19:2657-2663, 2000.

Behrens A., Sibilia M., David J.P. et al.: Impaired postnatal hepatocyte proliferation and liver regeneration in mice lacking c-jun in the liver. EMBO J. 21:1782-1790, 2002.

Behrens A., Sibilia M., Wagner E.F.: Aminoterminal phosphorylation of c-Jun regulates stress-induced apoptosis and cellular proliferation. Nat. Genet. 21:326-329, 1999.

Benbrook D.M., Jones N.C.: Heterodimer formation between CREB and JUN proteins. Oncogene 5:295-302, 1990.

Bengal E., Ransone L., Scharfmann R. et al.: Functional antagonism between c-Jun and MyoD proteins: a direct physical association. Cell 68:507-519, 1992.

Bernt C., Vennegeerts T., Beuers U. et al.: The human transcription factor AP-1 is a mediator of bile acid-induced liver cell apoptosis. Biochem. Biophys. Res. Commun. 340:800806, 2006.

Black E.J., Walker M., Clark W. et al.: Cell transformation by v-Jun deactivates ERK MAP kinase signalling. Oncogene 21:65406548, 2002.

Castellazzi M., Spyrou G., La Vista N. et al.: Overexpression of c-jun, junB, or junD affects cell growth differently. Proc. Natl. Acad. Sci. U.S.A. 88:8890-8894, 1991.

Cheung N.S., Carroll F.Y., Larm J.A. et al.: Kainate-induced apoptosis correlates with c-
Jun activation in cultured cerebellar granule cells. J. Neurosci. Res. 52:69-82, 1998.

Cooper S.J., Macgowan J., Ranger-Moore J. et al.: Expression of dominant negative c-jun inhibits ultraviolet B-induced squamous cell carcinoma number and size in an SKH-1 hairless mouse model. Mol. Cancer Res. 1: 848-854, 2003.

Dong Z., Crawford H.C., Lavrovsky V. et al.: A dominant negative mutant of jun blocking 12O-tetradecanoylphorbol-13-acetate-induced invasion in mouse keratinocytes. Mol. Carcinog. 19:204-212, 1997.

Eferl R., Sibilia M., Hilberg F. et al.: Functions of c-Jun in liver and heart development. J. Cell Biol. 145:1049-1061, 1999.

Elsasser A., Franzen M., Kohlmann A. et al.: The fusion protein AML1-ETO in acute myeloid leukemia with translocation $\mathrm{t}(8 ; 21)$ induces cjun protein expression via the proximal AP-1 site of the c-jun promoter in an indirect, JNKdependent manner. Oncogene 22:5646-5657, 2003.

Estus S., Zaks W.J., Freeman R.S et al.: Altered gene expression in neurons during programmed cell death: identification of c-jun as necessary for neuronal apoptosis. J. Cell Biol. 127:1717-1727, 1994.

Fialka I., Schwarz H., Reichmann E. et al.: The estrogen-dependent c-JunER protein causes a reversible loss of mammary epithelial cell polarity involving a destabilization of adherens junctions. J. Cell Biol. 132:11151132, 1996.

Finch S., Joseloff E., Bowden T.: JunB negatively regulates AP-1 activity and cell proliferation of malignant mouse keratinocytes. J. Cancer Res. Clin. Oncol. 128:3-10, 2002.

Florin L., Hummerich L., Dittrich B.T. et al.: Identification of novel AP-1 target genes in fibroblasts regulated during cutaneous wound healing. Oncogene 23:7005-7017, 2004.

Florin L., Knebel J., Zigrino P. et al.: Delayed wound healing and epidermal hyperproliferation in mice lacking JunB in the skin. J. Invest. Dermatol. 126:902-911, 2006.

Gaub M. P., Bellard M., Scheuer I. et al.: Activation of the ovalbumin gene by the estrogen receptor involves the fos-jun complex. Cell 63:1267-1276, 1990.

Grigoriadis A.E., Schellander K., Wang Z.Q., Wagner E.F.: Osteoblasts are target cells for transformation in c-fos transgenic mice. J. Cell Biol. 122:685-701, 1993.

Ham J., Babij C., Whitfield J. et al.: A c-Jun dominant negative mutant protects sympathetic neurons against programmed cell death. Neuron 14:927-939, 1995.

Hartl M., Reiter F., Bader A.G. et al.: JAC, a direct target of oncogenic transcription factor Jun, is 
involved in cell transformation and tumorigenesis. Proc. Natl. Acad. Sci. U.S.A. 98:13601-13606, 2001.

Hibi M., Lin A., Smeal T. et al.: Identification of an oncoprotein- and UV-responsive protein kinase that binds and potentiates the c-Jun activation domain. Genes Dev. 7:2135-2148, 1993.

Hilberg F., Aguzzi A., Howells N., Wagner E.F.: c-jun is essential for normal mouse development and hepatogenesis. Nature 365: 179-181, 1993.

Hilfiker-Kleiner D., Hilfiker A., Kaminski K. et al.: Lack of JunD promotes pressure overloadinduced apoptosis, hypertrophic growth, and angiogenesis in the heart. Circulation 112: 1470-1477, 2005.

Inagaki N., Maekawa T., Sudo T. et al.: c-Jun represses the human insulin promoter activity that depends on multiple cAMP response elements. Proc. Natl. Acad. Sci. U.S.A. 89: 1045-1049, 1992.

Jochum W., Passegue E., Wagner E.F.: AP-1 in mouse development and tumorigenesis. Oncogene 20:2401-2412, 2001.

Johnson R., Spiegelman B., Hanahan D., Wisdom R.: Cellular transformation and malignancy induced by ras require c-jun. Mol. Cell Biol. 16:4504-4511, 1996.

Johnson R.S., Van Lingen B., Papaioannou V.E., Spiegelman B. M.: A null mutation at the cjun locus causes embryonic lethality and retarded cell growth in culture. Genes Dev. 7: 1309-1317, 1993.

Jonat C., Rahmsdorf H.J., Park K.K., Cato A.C., Gebel S., Ponta H., Herrlich P.: Antitumor promotion and antiinflammation: downmodulation of AP-1 (Fos/Jun) activity by glucocorticoid hormone. Cell 62:1189-1204, 1990.

Kanamoto T., Mota M., Takeda K. et al.: Role of apoptosis signal-regulating kinase in regulation of the c-Jun $\mathrm{N}$-terminal kinase pathway and apoptosis in sympathetic neurons. Mol. Cell Biol. 20:196-204, 2000.

Karin M., Liu Z., Zandi E.: AP-1 function and regulation. Curr. Opin. Cell Biol. 9:240-246, 1997.

Kolbus A., Herr I., Schreiber M. et al.: C-Jundependent CD95-L expression is a ratelimiting step in the induction of apoptosis by alkylating agents. Mol. Cell. Biol. 20:575582, 2000.

Kouzarides T., Ziff E.: Leucine zippers of fos, jun and GCN4 dictate dimerization specificity and thereby control DNA binding. Nature 340: 568-571, 1989.

Leaner V.D., Kinoshita I., Birrer M.J.: AP-1 complexes containing cJun and JunB cause cellular transformation of Rat1a fibroblasts and share transcriptional targets. Oncogene 22:5619-5629, 2003.

Lee W., Mitchell P., Tjian R.: Purified transcription factor AP-1 interacts with TPA-inducible enhancer elements. Cell 49:741-52, 1987.

Liu Y., Ludes-Meyers J., Zhang Y. et al.: Inhibition of AP-1 transcription factor causes blockade of multiple signal transduction pathways and inhibits breast cancer growth. Oncogene 21: 7680-7689, 2002.

Lloyd A., Yancheva N., Wasylyk B.: Transformation suppressor activity of a Jun transcription factor lacking its activation domain. Nature 352:635-638, 1991.

Ludes-Meyers J.H., Liu Y., Munoz-Medellin D. et al.: AP-1 blockade inhibits the growth of normal and malignant breast cells. Oncogene 20:2771-2780, 2001.

Macian F., Lopez-Rodriguez C., Rao A.: Partners in transcription: NFAT and AP-1. Oncogene 20: 2476-2489, 2001.

MacLaren A., Clark W., Black E.J. et al.: v-Jun stimulates both cdk2 kinase activity and G1/S progression via transcriptional repression of p21 CIP1. Oncogene 22:2383-2395, 2003.

Maeno K., Masuda A., Yanagisawa K. et al.: Altered regulation of c-jun and its involvement in anchorage-independent growth of human lung cancers. Oncogene 25:271277, 2006.

Marshall G.M., Vanhamme L., Wong W.Y., Su H., Vogt P.K.: Wounding acts as a tumor promoter in chickens inoculated with avian sarcoma virus 17. Virology 188:373-377, 1992.

Marshall G. M., Vanhamme L.: Characterization of sarcoma cell lines from v-jun transgenic mice. Cancer Res. 53:622-626, 1993.

Naruishi K., Nishimura F., Yamada-Naruishi H., Omori K., Yamaguchi M., Takashiba S.: Cjun N-terminal kinase (JNK) inhibitor, SP600125, blocks interleukin (IL)-6-induced vascular endothelial growth factor (VEGF) production: cyclosporine A partially mimics this inhibitory effect. Transplantation 76:1380-1382, 2003.

Nead M.A., Baglia L. A., Antinore M.J., Ludlow J.W., McCance D.J.: Rb binds c-Jun and activates transcription. EMBO J. 17:23422352, 1998 .

Neyns B., Teugels E., Bourgain C. et al.: Alteration of jun proto-oncogene status by plasmid transfection affects growth of human ovarian cancer cells. Int. J. Cancer 82: 687-693, 1999.

Nishimura T., Vogt P.K.: The avian cellular homolog of the oncogene jun. Oncogene 3: 659-663, 1988.

Passegue E., Jochum W., Schorpp-Kistner M. et al.: Chronic myeloid leukemia with increased granulocyte progenitors in mice lacking junB 
expression in the myeloid lineage. Cell 104: 21-32, 2001.

Passegue E., Wagner E.F.: JunB suppresses cell proliferation by transcriptional activation of p16(INK4a) expression. EMBO J. 19:29692979, 2000.

Pfarr C.M., Mechta F., Spyrou G. et al.: Mouse JunD negatively regulates fibroblast growth and antagonizes transformation by ras. Cell 76:747-60, 1994.

Poindessous-Jazat V., Augery-Bourget Y., RobertLezenes J.: c-Jun modulates apoptosis but not terminal cell differentiation in murine erythroleukemia cells. Leukemia 16:233-243, 2002.

Qiao L., Han S.I., Fang Y. et al.: Bile acid regulation of $\mathrm{C} / \mathrm{EBPbeta}, \mathrm{CREB}$, and c-Jun function, via the extracellular signal-regulated kinase and c-Jun NH2-terminal kinase pathways, modulates the apoptotic response of hepatocytes. Mol. Cell. Biol. 23:3052-3066, 2003.

Rahmsdorf H.J: Jun: transcription factor and oncoprotein. J. Mol. Med. 74:725-747, 1996.

Raitano A.B., Halpern J.R., Hambuch T.M., Sawyers C.L.: The Bcr-Abl leukemia oncogene activates Jun kinase and requires Jun for transformation. Proc. Natl. Acad. Sci. U.S.A. 92:11746-11750, 1995.

Rangatia J., Vangala R.K., Singh S.M. et al.: c-Jun expression in acute myeloid leukemias inhibits $\mathrm{C} / \mathrm{EBPalpha}$ DNA binding via leucine zipper domain interaction. Oncogene 22:4760-4764, 2003.

Ricci R., Eriksson U., Oudit G.Y. et al.: Distinct functions of junD in cardiac hypertrophy and heart failure. Genes Dev. 19:208-213, 2005.

Rinehart-Kim J., Johnston M., Birrer M., Bos T.: Alterations in the gene expression profile of MCF-7 breast tumor cells in response to c-Jun. Int. J. Cancer 88:180-190, 2000.

Roffler-Tarlov S., Brown J.J., Tarlov E. et al.: Programmed cell death in the absence of c-Fos and c-Jun. Development 122:1-9, 1996.

Rossant J.: Mouse mutants and cardiac development: new molecular insights into cardiogenesis. Circ. Res. 78:349-353, 1996.

Schorpp-Kistner M., Wang Z.Q., Angel P., Wagner E.F.: JunB is essential for mammalian placentation. EMBO J. 18:934-948, 1999.

Schreiber M., Kolbus A., Piu F et al.: Control of cell cycle progression by c-Jun is p53 dependent. Genes Dev. 13: 607-619, 1999.

Schuh A.C., Keating S.J., Monteclaro F.S. et al.: Obligatory wounding requirement for tumorigenesis in v-jun transgenic mice. Nature 346:756-760, 1990.

Schüle R., Rangarajan P., Kliewer S. et al.: Functional antagonism between oncoprotein c-
Jun and the glucocorticoid receptor. Cell 62: 217-226, 1990.

Schütte J., Minna J.D., Birrer M.J.: Deregulated expression of human c-jun transforms primary rat embryo cells in cooperation with an activated c-Ha-ras gene and transforms rat-1a cells as a single gene. Proc. Natl. Acad. Sci. U.S.A. 86: 2257-2261, 1989.

Ševčíková S., Souček K., Kubala L. et al.: Differential effects of v-Jun and c-Jun proteins on v-myb-transformed monoblasts. Cell. Mol. Life Sci. 59:1690-1705, 2002.

Shaulian E., Karin M.: AP-1 in cell proliferation and survival. Oncogene 20:2390-2400, 2001.

Shirai K., Okada Y., Saika S., Senba E., Ohnishi Y.: Expression of transcription factor AP-1 in rat lens epithelial cells during wound repair. Exp. Eye Res. 73:461-468, 2001.

Smeal T., Binetruy B., Mercola D. et al.: Oncoprotein-mediated signalling cascade stimulates c-Jun activity by phosphorylation of serines 63 and 73. Mol. Cell. Biol. 12: 3507-3513, 1992.

Smeal T., Binetruy B., Mercola D.A. et al.: Oncogenic and transcriptional cooperation with Ha-Ras requires phosphorylation of c-Jun on serines 63 and 73. Nature 354:494-496, 1991.

Suto R., Tominaga K., Mizuguchi H. et al.: Dominant-negative mutant of c-Jun gene transfer: a novel therapeutic strategy for colorectal cancer. Gene Ther. 11:187-193, 2004.

Szabo E., Riffe M.E., Steinberg S.M., Birrer M.J., Linnoila R.I.: Altered cJUN expression: an early event in human lung carcinogenesis. Cancer Res. 56:305-315, 1996.

Szabowski A., Maas-Szabowski N., Andrecht S. et al.: c-Jun and JunB antagonistically control cytokine-regulated mesenchymal-epidermal interaction in skin. Cell 103:745-755, 2000.

Thepot D., Weitzman J.B., Barra J. et al.: Targeted disruption of the murine junD gene results in multiple defects in male reproductive function. Development 127:143-153, 2000.

Tsai E., Yie J., Thanos D., Goldfeld A.E.: Celltype-specific regulation of the human tumor necrosis factor alpha gene in B cells and $T$ cells by NFATp and ATF-2/JUN. Mol. Cell. Biol. 16:5232-5244, 1996.

Wang Z.Q., Liang J., Schellander K. et al.: c-fosinduced osteosarcoma formation in transgenic mice: cooperativity with c-jun and the role of endogenous c-fos. Cancer Res. 55:6244-6251, 1995.

Watts R.G., Ben-Ari E.T., Bernstein L.R. et al.: cjun and multistage carcinogenesis: association of overexpression of introduced c-jun with progression toward a neoplastic endpoint in mouse JB6 cells sensitive to tumor promoter- 
induced transformation. Mol. Carcinog. 13: 27-36, 1995.

Weitzman J.B., Fiette L., Matsuo K., Yaniv M.: JunD protects cells from p53-dependent senescence and apoptosis. Mol. Cell 6:11091119, 2000.

Wiggan O., Hamel P.A.: Pax3 regulates morphogenetic cell behavior in vitro coincident with activation of a $\mathrm{PCP} /$ noncanonical Wnt-signalling cascade. J. Cell Sci. 115:531-541, 2002.

Wilkinson D.G., Bhatt S., Ryseck R.P., Bravo R.: Tissue-specific expression of c-jun and junB during organogenesis in the mouse. Development 106:465-471, 1989.

Yang L., Kim H.T., Munoz-Medellin D. et al.: Induction of retinoid resistance in breast cancer cells by overexpression of cJun. Cancer Res. 57:4652-4661, 1997.

Yasumoto H., Kim S., Zhan Y. et al.: Negative cjun gene transfer inhibits vascular smooth muscle cell proliferation and neointimal hyperplasia in rats. Gene Ther. 8:1682-1689, 2001.

Young M.R., Li J.J., Rincon M. et al.: Transgenic mice demonstrate AP-1 (activator protein-1) transactivation is required for tumor promotion. Proc. Natl. Acad. Sci. U.S.A. 96: 9827-9832, 1999.

Zhou F., Thompson E.B.: Role of c-jun induction in the glucocorticoid-evoked apoptotic pathway in human leukemic lymphoblasts. Mol. Endocrinol. 10:306-316, 1996. 